\title{
Transport properties of non-Newtonian nanofluids and applications
}

\author{
R. Sivaraj ${ }^{1, a}$ and Santo Banerjee ${ }^{2, b}$ \\ ${ }^{1}$ Department of Mathematics, School of Advanced Sciences, Vellore Institute of Technology, Vellore 632014, India \\ 2 Department of Mathematical Sciences, Politecnico di Torino, Turin, Italy
}

Received 10 March 2021 / Accepted 12 March 2021 / Published online 21 May 2021

(C) EDP Sciences, Springer-Verlag GmbH Germany, part of Springer Nature 2021

The nanofluids have acquired substantial attention in the last few decades, due to the improved thermophysical properties such as density, viscosity, thermal conductivity, and specific heat capacity. From the literature, it is noticed that nanofluids find numerous applications in diverse areas including petroleum industry, lubrication, surface coating, biomedical, environmental remediation, and electronics cooling systems. This special issue comprises the theoretical/experimental research articles which elucidate the research efforts and recent developments on the transport properties of non-Newtonian nanofluids with the purpose to provide guidelines for future research directions. This issue consists of 29 manuscripts which are divided into six sections, namely, heat transfer of nanofluids in cavities and annulus, boundary layer analysis with nanofluids over different geometries, transport properties of nonNewtonian nanofluids over plates, dispersion and stability analysis of nanofluids, heat transfer in channels, and dynamics of nonlinear flows. These sections elucidate the impacts of several effects on transport characteristics of various nanofluids over different geometries. In this issue, the transport properties of various non-Newtonian nanofluids such as the Carreau nanofluid, Casson nanofluid, Jeffrey nanofluid, Maxwell nanofluid, Oldroyd-B nanofluid, power-law nanofluid, third-grade nanofluid, and Williamson nanofluid are presented graphically and the results are discussed with an adequate physical interpretation.

Section I of this issue summarises the results on heat transfer of nanofluids in cavities and annular. Aly et al. [1] analysed the natural convection flow resulting from rotating circular cylinders in a horizontal wavy cavity fully filled by a nanofluid. An incompressible smoothed particle hydrodynamics method is used to examine the horizontal wavy cavity which is saturated with three layers of heterogeneous porous media. The results revealed that an increase in the radius of the inner cylinders enhances the temperature distributions and the

\footnotetext{
${ }^{a}$ e-mail: sivaraj.kpm@gmail.com

b e-mail: santoban@gmail.com (corresponding author)
}

nanofluid flow. Growth of the paddle lengths enhances the velocity field in all the porous layers. Ahmed et al. [2] applied the finite volume method based on the SIMPLE algorithm to an investigation of the convective flow of a power-law nanofluid within a cubical enclosure saturated with a homogeneous/heterogeneous porous medium in the presence of a heat source. Three different levels of heterogeneous porous media and three values of power-law indices are investigated. The obtained results indicate that with a homogeneous porous medium and a Newtonian fluid, the temperature and nanoparticle volume fraction distributions increase inside the cubic enclosure. Gokulavani and Muthtamilselvan [3] numerically examined the influence of the thermal radiation on the free convective flow of water-based nanodiamondnickel nanocomposite in a multiple port open cavity. One inlet port and two outlet ports are situated on the perpendicular walls and the remaining walls of the cavity are considered as adiabatic. The heated thin baffle is located inside the cavity. An alternate direction implicit scheme-based finite difference process is used for discretization of the governing equations. The results manifest that the average heat transfer rate reduces with the baffle placed far from the inlet. An enhancement in the radiation effect inside the cavity results in increasing the thermal performance of the cavity. Reddy et al. [4] utilized the implicit finite difference method-based numerical scheme to study the effect of an axially varying temperature in the form of sinusoidal thermal profiles along the side walls of an annular enclosure containing different hybrid nanofluids with insulated horizontal boundaries. It is clear from this study that the phase deviation is a crucial parameter in enhancing or suppressing the thermal transport in the annulus. For a particular choice of the phase deviation, it is possible to increase the flow strength and the associated thermal dissipation rate. The simulations reveal that the copper nanofluid or the hybrid nanofluid combined with copper could increase the thermal transport.

Section II of this issue elucidates the boundary layer analysis with nanofluids over different geometries. Naveenkumar et al. [5] scrutinized the influence of acti- 
vation energy over a Darcy-Forchheimer flow of a Casson hybrid nanofluid. The flow is caused by a vertically upward/downward moving rotating disk. The numerical solution is attained by using the Runge-KuttaFehlberg method. The obtained results reveal that an increase in the Casson parameter reduces the velocity of the fluid. The increassing values of the expansion/contraction parameter increase the velocity, thermal and concentration profiles. Waqas et al. [6] utilized the bvp4c MATLAB solver to study the effects of MHD, thermal radiation, activation energy, and Wu's slip on bio-convection flow of the Oldroyd-B fluid over a stretching cylinder/plate in the presence of swimming motile microorganisms. It is noticed from the results that an increment in the magnetic parameter and the first order velocity-slip parameter magnitudes diminishes the velocity field. The temperature of the OldroydB fluid dwindles for higher Deborah number with the retardation time parameter. Waqas et al. [7] investigated the influences of nonlinear convection, radiation, dual stratifications, Brownian motion and thermophoresis effects on the magneto-Jeffrey nanomaterial flow by utilizing the homotopy method. It is noticed from the results that temperature and concentration of the fluid diminish for higher stratification factors. An increase in the radiation factor efficiently amplifies the temperature of the system. Hanif et al. [8] analysed the effects of thermal radiation, external magnetic field, and non-uniform heat source/sink on the transport properties of a water-based hybrid nanofluid flow with suspension of hybrid nanoparticles $\left(\mathrm{Cu}-\mathrm{Fe}_{3} \mathrm{O}_{4}\right)$ past a vertical cone saturated with a porous medium by using the finite difference method. It is detected that the Nusselt number upsurges for growing nanoparticle concentrations in the fluid. For nanofluids and hybrid nanofluids, one finds that the temperature of the system increases when thermal radiation and a magnetic field are present. Samrat et al. [9] scrutinized the magnetohydrodynamic free convection flow along the higher part of the paraboloid of revolution. It is found that the magnetic field signifies an additional conflicting force to the fluid motion. The impact of the Brownian moment is higher on a non-Newtonian flow compared with Newtonian flow. Accelerating the strength of the magnetic field and the Eckert number reduces the heat transfer rate. Revathi et al. [10] investigated a binary chemical nanofluid dissipative flow over a curved stretching sheet with activation energy subject to a convective boundary condition. This investigation reveals that the reaction rate parameter and the temperature difference parameter are helpful to ameliorate the mass transfer rate. The concentration is enhanced for higher values of the activation energy variable. A heat transfer rate enhancement is observed with the influence of the heat transfer Biot number.

Section III of this issue examines the transport properties of non-Newtonian nanofluids over various plates. Buongiorno [11] has analysed seven slip mechanisms such as Brownian diffusion, inertia, thermophoresis, Magnus effect, diffusiophoresis, fluid drainage, and gravity, and determined that the important slip mech- anisms in nanofluids are thermophoresis and Brownian diffusion. Based on this finding, many researchers have adopted various non-Newtonian fluid models to analyse the transport properties of non-Newtonian nanofluids. Loganathan et al. [12] inspected the impacts of MHD, non-Fourier heat and mass flux, velocity slip, Newtonian heating, and thermal radiation on the DarcyForchheimer flow of a third-grade nanofluid over an elastic sheet. A series solutions is derived by adopting a homotopic simulation and the entropy minimization of the total system is calculated. The results indicate that the velocity of the fluid gradually decreases for positive values of the porosity parameter and the Forchheimer number. An increase in Forchheimer number reduces the entropy generation rate. Temperature profiles intensify for higher values of the radiation parameter. Waqas et al. [13] presented a mathematical model to scrutinize the aspect of Cattaneo-Christov heat and mass flux, swimming motile microorganisms, activation energy and double stratification effects on a steady 3D convectional flow of a Carreau nanofluid towards a bidirectional stretching surface. Numerical solutions are determined by using the collocation finite difference technique and a three-stage LobattoIIIa formula. A parametric analysis reveals that the nanoparticle temperature grows with an upsurge in the thermal stratification Biot number and temperature ratio parameter. The temperature profiles diminish for higher thermal relaxation time parameter. Meenakumari et al. [14] emphasized the effects of viscous dissipation, inclined magnetic field, radiation, chemical reaction, and non-uniform heat source/sink on a Williamson nanoliquid flow through a porous plate by employing the Runge-Kutta-Fehlberg-based shooting technique. Diverse nanoparticles including copper, silver, aluminium oxide, magnesium oxide, and titanium oxide are studied in this analysis. This analysis reports that aluminium oxide nanoliquid has the fastest fluid flow because aluminium oxide nanoliquid has the least density among the considered nanoparticle-based nanoliquids. Copper nanoliquid has the highest heat transfer characteristics because copper has the highest thermal conductivity among the considered nanoparticles. Amplifying the viscous dissipation renders the higher heat transfer. Reddy and Lakshminarayana [15] adopted the Runge-Kutta-Fehlberg-based shooting technique to investigate the three-dimensional magnetohydrodynamic flow of an upper convected Maxwell nanofluid with chemical reaction, thermal radiation, cross-diffusion and heat source effects along a stretching sheet. It is noticed that increasing the Dufour and thermal radiation parameters decreases the temperature field and increases the concentration field. The growing values of the Deborah number and the magnetic field reduce the friction factor, and the Nusselt and Sherwood numbers. Amplifying the heat source and chemical reaction parameters reduces the Nusselt number and boosts the Sherwood number. Sandhya et al. [16] studied the chemical reaction, radiation, and viscous dissipation on a magnetohydrodynamic radiative Casson nanoliquid thin film flow generated by stretching sheet 
by using the Runge-Kutta-Fehlberg method. It is evident from the results that stronger magnetic fields cause the velocity in the thin film to decline; meanwhile a converse impact on the free surface temperature is noticed. Higher values of unsteady, magnetic and Casson parameters decrease the size of film thickness. Ghatani et al. [17] investigated the flow of thin Casson nanoliquid film over a plate with suction/injection and a transverse magnetic field. The perturbation method is utilized to obtain the velocity and temperature distributions. The Runge-Kutta method is adopted to solve the nonlinear film evolution equations. It is observed that the liquid film thickness is increased with Hartmann number, nanoparticle volume fraction, porosity parameter, and Casson parameter. The rate of film thinning increases for suction whereas the reverse phenomenon is found for injection. Zhang et al. [18] studied the heat transfer and flow behaviours of ethylene vinyl acetate nanofluid film by tape casting. A power-law velocity-slip model, which is well suited for describing the slip characteristics of polymer films, is presented. The analytical solutions are obtained by utilizing a differential transform method with the Newton iteration method. It is noteworthy that the heat transfer rate of the power-law fluid is weaker than that of the Newtonian fluid. The temperature reduces with enhancement in the temperature control parameter and the convective heat transfer coefficient.

Section IV of this issue presents a dispersion and stability analysis of various nanofluids. Chaturvedi and Sharma [19] examined the dispersion regimes present in single-step silica nanofluids under varying conditions viz., $\mathrm{pH}(2-12)$ and salinity $(0-4 w t \% \mathrm{NaCl})$. Initially, based on the ratios of viscosity, electrical conductivity, UV-vis absorbance peak, and $\mathrm{CO}_{2}$ absorption, the presence of four dispersion regimes (well dispersed, partially agglomerated, fully agglomerated, and fully settled) were established in the nanofluid, which was further confirmed by SEM analysis. The results disclose that the nanofluid lacked any improvement and was similar to the base fluid in the fully settled regime. The formulated single-step silica nanofluids show an excellent dispersion behaviour over a wide range of salt and $\mathrm{pH}$ environments, which makes them highly viable candidates for improved carbon transport and storage applications in complex reservoirs. Ausaru et al. [20] investigated the impact of external body acceleration dispersion of a solute in non-Newtonian fluid flow in the presence of the solute absorption at the tube wall by using the finite-difference scheme. The pulsatile pressure gradient, Newtonian rheology, wall absorption, and external body acceleration on the mean concentration and dispersion coefficient are examined. Results indicate that the body acceleration affects the dispersion coefficient. Though the body acceleration is present, an upsurge in wall absorption coefficient reduces the mean concentration. Meghana et al. [21] analysed the effects of sinusoidal (sine wave) and non-sinusoidal (square wave, triangular wave, and sawtooth wave) forms of gravity modulation on Rayleigh-Benard convection in a ferromagnetic fluid and a nanoliquid with couple stress using linear and nonlinear stability analyses. From the linear analysis, the stability of the system is investigated by calculating the correction Rayleigh number using the Venezian method. The heat transfer coefficient is obtained by solving the non-autonomous Lorenz equation. It is observed that the couple stress parameter stabilizes the system and decreases the heat transfer. It is found that the square type of gravity modulation facilitates more heat transport than other types of gravity modulations. The higher concentration of alumina is shown to be preferable to the rather expensive copper nanoparticles in obtaining the same quantity of heat transfer. Awasthi et al. [22] examined the stability of the interface formed by a viscoelastic nanofluid and viscous incompressible fluid. The layout is taken in such a way that the viscoelastic nanofluid lies above the viscous fluid and, therefore, the interface experiences a Rayleigh-Taylor type instability. A power-law viscoelastic liquid is considered for the linear stability analysis and the potential flow theory of viscous fluids is enforced to solve the mathematical equations. An algebraic equation of the second degree is achieved and stability/instability is discussed based on the negative/positive roots of this equation. It is found that the shear-thinning fluid is more stable than the Newtonian fluid but the shear thickening fluid is more unstable. $\mathrm{Al}_{2} \mathrm{O}_{3}+\mathrm{H}_{2} \mathrm{O}$ nanofluid leads to the most stable configuration, while the most unstable interface is formed by $\mathrm{C} u O+\mathrm{E} G$. Gupta et al. [23] scrutinized the convective instability of blood with nanoparticles using the Casson fluid model. Small disturbances are added to the solution and the normal mode technique is used to convert partial differential equations into ordinary differential equations. Furthermore, these equations are solved using the one term Galerkin method for freefree, rigid-free and rigid-rigid boundaries. The complex expressions of the Rayleigh number are simplified with valid approximations to get analytical results. The commonly used metals (copper, silver, gold, and iron) and non-metals (alumina, silica, copper oxide, and titanium oxide) in nano drug delivery systems are taken to investigate their impact on the instability of blood flow. The results indicate that the alumina nanoparticles have a more destabilizing impact on blood than copper and could play a significant role on human health, more specifically in the cardiovascular system. The stability pattern followed by metallic nanofluids is copper-blood $>$ silver-blood $>$ gold-blood $>$ iron-blood and for non-metallic nanofluids it is alumina-blood> silica-blood > copper oxide-blood $>$ titanium oxide-blood.

Section V of this issue analyses the heat transfer characteristics of various fluids in different channels. Magesh and Kothandapani [24] explored the characteristics of peristaltic flow of a Johnson Segalman fluid in an asymmetric curved channel by using the perturbation technique. The exceedingly nonlinear governing equations are relatively simplified by adopting the assumptions of long wavelength and small Reynolds number approximations. It is noticed that the efficiency of the peristaltic pumping is deteriorated with greater values of the curvature parameter, which evidently indicates that 
the performance of the pumping induced by the peristalsis can be controlled even by adjusting the curvature of the channel. The temperature of the fluid flow within the channel grown by increasing the curvature parameter and the Weissenberg Number, whereas the converse behaviour is found with respect to the Brinkman number. Kumar and Srinivas [25] numerically scrutinized the pulsating flow of a hydromagnetic nanofluid in a channel with Joule heating and velocity slip. Blood is considered as a base fluid that is non-Newtonian, and $\mathrm{Al}_{2} \mathrm{O}_{3}$ is considered as a nanoparticle. It is found that the effects of Joule heating and velocity slip significantly affect blood $-\mathrm{Al}_{2} \mathrm{O}_{3}$ flow in the vertical channel. As the Hartmann number increases, the temperature increases at the suction wall and decreases at the injection wall. In the presence of slip, the Nusselt number is $2 \%$ higher at the injection wall than under the nonslip condition. Venkatesan and Reddy [26] adopted the perturbation method to study the influence of pulsating MHD flow of the $\mathrm{Al}_{2} \mathrm{O}_{3}$-blood nanoliquid between parallel flat plates with thermal radiation. The rheological behaviour of blood is modelled using the Oldroyd-B fluid model. The results reveal that an upsurge in heat source increases the nanoliquid temperature whereas the nanoliquid temperature decreases for higher radiation parameter. The heat transfer enhances for higher frequency parameter. An upsurge in viscous dissipation and nanoparticle volume fraction boosts the heat transfer rate. Mahanthesh and Thriveni [27] examined the impacts of the exponential heat source linked to space, nonlinear thermal radiation, inclined magnetic force, variable thermal conductivity and variable viscosity on the nano-bioconvective flow between two turntables by using the finite difference method. An optimization procedure is implemented via the response surface methodology for effective parameters such as the thermophoresis parameter, radiation parameter and Hartmann number on the heat transfer rate. It is noteworthy that the axial velocity is a dwelling function of the inclined angle of the magnetic field, and the variable viscosity parameter. The heat transport rate is highly sensitive on the nonlinear thermal radiation parameter, compared to the thermophoresis effect and Hartmann number.

Section VI of this issue scrutinizes the dynamics of nonlinear flows. Pradhan et al. [28] proposed a fourdimensional conservative dynamical system to investigate ion-acoustic wave flow in a dense quantum plasma containing positive beam ions, positive ions and electrons. The equilibrium points of the dynamical system and its stability are briefly discussed and the dynamics of chaotic features for ion-acoustic waves are analysed by considering the effects of Mach number and quantum diffraction parameter. It is observed that phase projections, graphs of maximum Lyapunov exponents, basins of attraction and bifurcation diagrams have supported the coexisting features for ion-acoustic waves. The multistability phenomenon for ion-acoustic waves without the introduction of an external force is elucidated. He et al. [29] presented an analysis framework to gain understanding of the dynamics of a two-dimensional nonautonomous coronary artery model. The analysis shows that the coronary artery system exhibits chaotic attractors, quasi-periodic, and periodic orbits. It is noteworthy that the presence of coexisting attractors reflect the high sensitivity of the system. The coexisting bifurcation model and phase spaces reveal that the coronary artery system with two different sets of initial conditions produces two 2-cycles, symmetric chaotic attractors, and quasi-periodic and chaotic attractors. Complexity simulation analysis demonstrates that the coexisting attractors exhibit different complexity values. Consequently, the dynamic characteristics of the coronary artery system are difficult to predict. Yan et al. [30] inspected the dynamical changes and its corresponding phase space complexity in a stochastic red blood cell system. The system is obtained by incorporating power noise with the associated sinusoidal flow. The dynamical complexity of the sinusoidal flow in the system is investigated by a heterogeneous recurrencebased entropy. It is noticed that an very similar kind of transaction in the trajectories is found in periodic states and in the chaotic states for sinusoidal blood flow. The quantification has successfully classified the stochastic blood flow system to a high degree. In addition, more complex and richer dynamics has been quantified in the stochastic red blood cell system than with its deterministic part.

We would like to express our sincere thanks to all the editorial members of the European Physical Journal Special Topics for their motivation and support. We thank the reviewers for their valuable comments and suggestions which significantly helps to improve the quality of the manuscripts. We also extend our profound gratitude to all the authors for contributing their research articles which address the recent developments and research efforts on nanofluids with the purpose of providing guidelines for future research directions.

\section{References}

1. A.M. Aly, Z.A.S. Raizah, S.E. Ahmed, ISPH simulations of natural convection from rotating circular cylinders inside a horizontal wavy cavity filled with a nanofluid and saturated by a heterogeneous porous medium. Eur. Phys. J. Spec. Top. (2021). https://doi.org/10.1140/ epjs/s11734-021-00050-y

2. S.E. Ahmed, Z.A.S. Raizah, A.M. Aly, Three dimensional flow of a power-law nanofluid within a cubic domain filled with a heat generating and 3D-heterogeneous porous medium. Eur. Phys. J. Spec. Top. (2021). https://doi.org/10.1140/epjs/ s11734-021-00040-0

3. P. Gokulavani, M. Muthtamilselvan, Radiation effect of ND Ni nanocomposite, water filled multiport cavity with heated baffle. Eur. Phys. J. Spec. Top. (2021). https:// doi.org/10.1140/epjs/s11734-021-00047-7

4. N.K. Reddy, H.A.K. Swamy, M. Sankar, Buoyant convective ow of different hybrid nanoliquids in a non-uniformly heated annulus. Eur. Phys. J. Spec. Top. (2021). https://doi.org/10.1140/epjs/ s11734-021-00034-y 
5. R. Naveenkumar, R.J.P. Gowda, B.J. Gireesha, B.C. Prasannakumara, Non-Newtonian hybrid nanofluid flow over vertically upward/downward moving rotating disk in a Darcy-Forchheimer porous medium. Eur. Phys. J. Spec. Top. (2021). https://doi.org/10.1140/epjs/ s11734-021-00054-8

6. H. Waqas, S.A. Khan, M. Alghamdi, M.S. Alqarni, T. Muhammad, Numerical simulation for bio-convection flow of magnetized non- Newtonian nanofluid due to stretching cylinder/plate with swimming motile microorganisms. Eur. Phys. J. Spec. Top. (2021). https://doi.org/10.1140/epjs/s11734-021-00041-z

7. M. Waqas, Z. Asghar, W.A. Khan, Thermo-solutal Robin conditions significance in thermally radiative nanofluid under stratification and magnetohydrodynamics. Eur. Phys. J. Spec. Top. (2021). https://doi. org/10.1140/epjs/s11734-021-00044-w

8. H. Hanif, I. Khan, S. Shafie, A novel study on hybrid model of radiative $\mathrm{Cu}-\mathrm{Fe} 3 \mathrm{O} 4$ water nanofluid over a cone with PHF/PWT. Eur. Phys. J. Spec. Top. (2021). https://doi.org/10.1140/epjs/s11734-021-00042-y

9. S.P. Samrat, N. Sandeep, M. Pasupula, Buoyancy effect on magnetohydrodynamic radiative flow of Casson fluid with Brownian moment and thermophoresis. Eur. Phys. J. Spec. Top. (2021). https://doi.org/10. 1140/epjs/s11734-021-00043-x

10. G. Revathi, V.S. Sajja, C.S.K. Raju, M.J. Babu, Numerical simulation for Arrhenius activation energy on the nanofluid dissipative flow by a curved stretching sheet. Eur. Phys. J. Spec. Top. (2021). https://doi.org/10. 1140/epjs/s11734-021-00048-6

11. J. Buongiorno, Convective transport in nanofluids. ASME J. Heat Transfer 128, 240-250 (2006)

12. K. Loganathan, N. Alessa, K. Tamilvanan, F.S. Alshammari, Significances of Darcy-Forchheimer porous medium in third-grade nanofluid flow with entropy features. Eur. Phys. J. Spec. Top. (2021). https://doi.org/ 10.1140/epjs/s11734-021-00056-6

13. H. Waqas, M. Imran, M.M. Bhatti, Bioconvection aspects in non-Newtonian three-dimensional Carreau nanofluid flow with Cattaneo-Christov model and activation energy. Eur. Phys. J. Spec. Top. (2021). https:// doi.org/10.1140/epjs/s11734-021-00046-8

14. R. Meenakumari, P. Lakshminarayana, K. Vajravelu, Unsteady MHD flow of a Williamson nanofluid on a permeable stretching surface with radiation and chemical reaction effects. Eur. Phys. J. Spec. Top. (2021). https://doi.org/10.1140/epjs/s11734-021-00039-7

15. M.V. Reddy, P. Lakshminarayana, Cross diffusion and heat source effects on a three-dimensional MHD flow of Maxwell nanofluid over a stretching surface with chemical reaction. Eur. Phys. J. Spec. Top. (2021). https:// doi.org/10.1140/epjs/s11734-021-00037-9

16. G. Sandhya, K. Malleswari, G. Sarojamma, K. Sreelakshmi, P.V.S. Narayana, Unsteady Casson nanofluid thin film flow over a stretching sheet with viscous dissipation and chemical reaction. Eur. Phys. J. Spec. Top. (2021). https://doi.org/10.1140/epjs/s11734-021-00033-z

17. Y. Ghatani, R. Singh, T.S. Maity, Unsteady thin Casson-nanoliquid film flow over a porous stretching sheet. Eur. Phys. J. Spec. Top. (2021). https://doi.org/ 10.1140/epjs/s11734-021-00055-7
18. Y. Zhang, Y. Zhang, B. Yuan, Y. Bai, Flow and heat transfer analysis of ethylene vinyl acetate nanofluid film in the process of tape casting. Eur. Phys. J. Spec. Top. (2021). https://doi.org/10.1140/epjs/ s11734-021-00058-4

19. K.R. Chaturvedi, T. Sharma, Investigating dispersion regimes for effective mass transfer in single-step silica nanofluids for improved CO2 utilization. Eur. Phys. J. Spec. Top. (2021). https://doi.org/10.1140/epjs/ s11734-021-00049-5

20. A. Ausaru, P. Nagarani, B.T. Sebastian, Dispersion with wall absorption in non-Newtonian fluid flow subjected to external body acceleration. Eur. Phys. J. Spec. Top. (2021). https://doi.org/10.1140/epjs/ s11734-021-00051-x

21. J. Meghana, S. Pranesh, P.G. Siddheshwar, Individual effects of sinusoidal and non-sinusoidal gravity modulation on Rayleigh-Benard convection in a ferromagnetic liquid and in a nanoliquid with couple stress. Eur. Phys. J. Spec. Top. (2021). https://doi.org/10.1140/ epjs/s11734-021-00036-w

22. M.K. Awasthi, Z. Uddin, R. Asthana, Temporal instability of a power-law viscoelastic nanofluid layer. Eur. Phys. J. Spec. Top. (2021). https://doi.org/10.1140/ epjs/s11734-021-00038-8

23. U. Gupta, J. Sharma, M. Devi, Double-diffusive instability of Casson nanofluids with numerical investigations for blood-based fluid. Eur. Phys. J. Spec. Top. (2021). https://doi.org/10.1140/epjs/s11734-021-00053-9

24. A. Magesh, M. Kothandapani, Heat and mass transfer analysis on non-Newtonian fluid motion driven by peristaltic pumping in an asymmetric curved channel. Eur. Phys. J. Spec. Top. (2021). https://doi.org/10.1140/ epjs/s11734-021-00035-x

25. P.B. Kumar, S. Srinivas, A note on the pulsatile flow of hydromagnetic Eyring-Powell nanofluid through a vertical porous channel. Eur. Phys. J. Spec. Top. (2021). https://doi.org/10.1140/epjs/s11734-021-00057-5

26. G. Venkatesan, A.S. Reddy. Insight into the dynamics of blood conveying alumina nanoparticles subject to Lorentz force, viscous dissipation, thermal radiation, Joule heating, and heat source. Eur. Phys. J. Spec, Top. (2021). https://doi.org/10.1140/epjs/ s11734-021-00052-w

27. B. Mahanthesh, K. Thriveni, Significance of inclined magnetic field on nano-bioconvection with nonlinear thermal radiation and exponential space based heat source: a sensitivity analysis. Eur. Phys. J. Spec. Top. (2021). https://doi.org/10.1140/epjs/ s11734-021-00045-9

28. B. Pradhan, A. Saha, H. Natiq, Multistability and dynamical properties of quantum ion-acoustic flow. Eur. Phys. J. Spec. Top. (2021). https://doi.org/10.1140/ epjs/s11734-021-00059-3

29. S. He, H. Natiq, S. Mukherjee, Multistability and chaos in a noise-induced blood flow. Eur. Phys. J. Spec. Top. (2021). https://doi.org/10.1140/epjs/ s11734-021-00032-0

30. B. Yan, S. Mukherjee, A. Saha, Exploring noise-induced chaos and complexity in a red blood cell system. Eur. Phys. J. Spec. Top. (2021). https://doi.org/10.1140/ epjs/s11734-021-00030-2 